
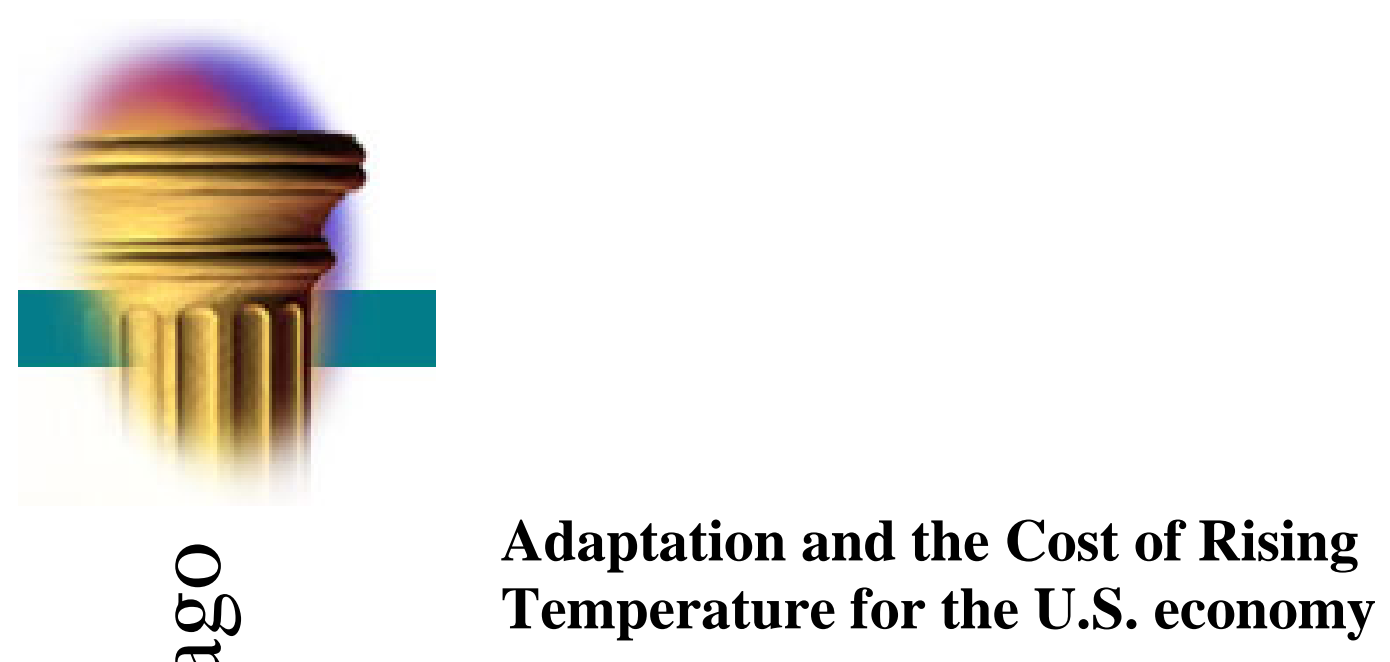

François Gourio and Charles Fries

\title{
March 2020
}

WP 2020-08

https://doi.org/10.21033/wp-2020-08

${ }^{*}$ Working papers are not edited, and all opinions and errors are the responsibility of the author(s). The views expressed do not necessarily reflect the views of the Federal Reserve Bank of Chicago or the Federal Reserve System. 


\title{
Adaptation and the Cost of Rising Temperature for the U.S. economy*
}

\author{
François Gourio $^{\dagger} \quad$ Charles Fries ${ }^{\ddagger}$
}

March 2020

\begin{abstract}
How costly will rising temperature due to climate change be for the U.S. economy? Recent research has used the well-identified response of output to weather to estimate this cost. But agents may adapt to the new climate. We propose a methodology to infer adaptation technology from the heterogeneous responses of output to weather observed currently across the U.S. Our model estimates how much each region has adapted already, and can predict how much each will adapt further after climate change. The size and distribution of losses from climate change vary substantially once adaptation is taken into account.
\end{abstract}

\section{Introduction}

Climate change will lead to rising temperature with myriad effects on humans. In this paper we focus on the effect on economic output, or income. How costly will rising temperature be for the U.S. economy? This question is critical to evaluate the appropriateness of policies designed to limit climate change.

The costs of climate change are represented in climate models (for instance, integrated assessment models (IAM)) through a "damage function". Early work relied on technological relationships, estimating the costs from an engineering perspective. This approach has the drawback that it is difficult to assemble detailed technological knowledge for all sectors of modern economies. As a result, critics of climate models (e.g., Pindyck [2013]) have emphasized the uncertainty in the damage function. A recent promising approach to estimate damages uses statistical methods to infer the effect of temperature from quasi-random weather variation. ${ }^{1}$ This literature builds on the

\footnotetext{
*We thank participants in presentations at Arizona State University, the Chicago Fed, and SED 2018, and in particular Gadi Barlevy, Jeff Campbell, Tatyana Deryugina, Olivier Deschenes, Stephie Fried, Dana Kiku, and Sam SchulhoferWohl for their comments or suggestions on earlier versions of this work. The views expressed here are those of the authors and do not necessarily represent those of the Federal Reserve Bank of Chicago or the Federal Reserve System.

${ }^{\dagger}$ Federal Reserve Bank of Chicago; 230 S. LaSalle Street, Chicago IL 60604. Email: francois.gourio@chi.frb.org

$\ddagger$ Columbia University, Law School. 435 W 116th St, New York, NY 10027. Email: Charles.Fries@columbia.edu

${ }^{1}$ For instance, see Hsiang et al. [2017] or Schlenker and Roberts [2009]. These estimates have been widely reported in national media (see for instance Plumer and Popovich [2017])
} 
recent "new climate-economy literature" (Dell et al. [2014]), which finds that higher temperature leads to lower income, even in wealthy economies such as the United States. ${ }^{2}$

The estimates in this literature, however, only capture the response to temporarily higher temperature, i.e. "weather". But the response to a permanent increase in temperature - such as that induced by climate change - might be quite different: agents would likely adapt to the higher temperature by changing their behavior. There is little agreement, however, on the magnitude of this adaptation response.

In this paper, we show how one can use the well-identified reduced-form estimates of the recent "new climate-economy" literature to infer the adaptation technology. We then illustrate the implications for predicting income losses from climate change. We find that taking adaptation into account significantly changes the magnitude, distribution, and location of losses.

How are we able to infer adaptation costs? Our starting point is the simple observation that the sensitivity of income to temperature is widely different within the United States today. Most authors focus on estimating "the" sensitivity of income to temperature, yet across U.S. counties this sensitivity varies by a factor of at least 10 .

What determines a county's sensitivity? Unsurprisingly, we show that climate - measured, for instance, simply as the county's long-run average temperature - explains most of this heterogeneity. Places that are ordinarily warm, such as the South, are nearly unaffected by high temperature realizations, while colder regions in the North exhibit larger sensitivities. This is the precise pattern one would expect from adaptation. Households and firms operating in the South are aware of their environment, and consequently, have made decisions to reduce the effect of high temperature. The North faces different conditions, and thus made different choices, despite having access to the same adaptation technology. Presumably, the cost of adapting to hot temperature cannot be justified given the cooler Northern climate.

Our key insight is that the reduced form estimates allow us to infer the benefit to adaptation in the North: the benefit is the product of the incidence of high temperature (e.g., the number of hot days per year), times the reduction in the cost of high temperature, i.e. the difference between the sensitivity of income to temperature in the North and the South. This is how much annual loss the North could save by adopting the South technology; hence, the cost of adaptation must be at least as large. While this seems to provide only a bound, note that we observe many regions, rather than just two; if each region chooses its level of adaptation optimally given its climate, we can recover the actual marginal cost of adaptation.

We implement this idea using a simple economic model of adaptation which incorporates (i) random temperature variation, (ii) economic output is sensitive to temperature, and (iii) an exante costly adaptation choice. Using indirect inference, we estimate the key parameters of the model - in particular, those that govern the adaptation technology - to fit the U.S. cross-section of sensitivities to temperature.

We then use our model to predict income losses from climate change. We find it useful to

\footnotetext{
${ }^{2}$ See for instance Dell et al. [2012], Deryugina and Hsiang [2014], Burke et al. [2015], and Colacito et al. [2019].
} 
compare the predictions of the model under two different assumptions about adaptation. First, we predict losses assuming that adaptation does not change, i.e. remains at the level chosen today given the current climate. Second, we predict losses assuming that adaptation is adjusted (optimized) given the new climate. We call these the fixed and endogenous adaptation cases respectively.

We obtain four main conclusions. First, and unsurprisingly, endogenous adaptation results in lower economic losses than if adaptation is fixed. For instance, in a severe climate change scenario (the Representative Concentration Pathway (RCP) 6.0 scenario, corresponding to a temperature increase of $5.1^{\circ} \mathrm{C}$ by year 2100), the median county loses about $2.9 \%$ of consumption (i.e., income net of adaptation costs) in the fixed adaptation scenario, but only about $1.8 \%$ in the endogenous adaptation scenario.

Second, we find that the dispersion in losses across U.S. counties is much smaller once adaptation is taken into account: the standard deviation of losses is $1.5 \%$ with fixed adaptation and $0.65 \%$ with endogenous adaptation. This is because adaptation allows the counties that have the most to lose from climate change to limit their losses.

Third, adaptation is more important when climate changes more: the difference between the median loss in the fixed and endogenous adaptation is only $0.14 \%$ in a mild climate change scenario (RCP 2.6), but rises to $3.45 \%$ in the most severe scenario (RCP 8.5).

Fourth, and perhaps most strikingly, the location of losses in the U.S. depends significantly on adaptation. Some authors assume that the same sensitivity holds across the entire United States, and that this sensitivity will remain fixed in the future. This implies that the South will suffer dramatic losses (for instance, see Hsiang et al. [2017]). In contrast, our fixed adaptation model, which leads to heterogeneous sensitivities across the U.S., suggests that the Midwest may be more affected than the South. This is because the South already has a low sensitivity today. This result also holds with endogenous adaptation.

This paper is, in part, a methodological contribution. While simple, the argument that heterogeneity in observed sensitivities can be used to estimate a structural model of adaptation, has not, to our knowledge, been exploited in the literature. The model is deliberately kept simple, but it can be extended to incorporate many additional realistic features of climate and the economy.

We do not take a stand on the exact mechanisms through which temperature reduces output; as we discuss below in more detail, a variety of mechanisms have been studied in the literature. We take a holistic (or macroeconomic) perspective and focus on the response of total income, without trying to decompose between the different margins at work, something we leave for future research.

We also do not attempt to directly measure adaptation investments in the data. First, our method does not require us to measure these investments, since we argue they can be inferred from observed behavior. Second, these investments likely take many forms, making them difficult to comprehensively measure: installing air conditioning (including in warehouses or factories); growing crops that are heat-resistant; building plants and infrastructure that can withstand extreme 
temperatures; and so on.

Finally, we emphasize three limitations of our study. First, we focus on the effect of temperature on income, and abstract from many phenomena linked to climate change and which imply additional economic costs, such as extreme weather events, sea level rise, changing precipitation patterns, etc. These phenomena could in principle be incorporated in our analysis, with the appropriate data and modeling. Second, our approach allows to recover the currently available adaptation technology; but this technology may change in the future. Third, our method requires some extrapolation outside the range of data observed today; we discuss this in section 4.3.

The rest of the paper is organized as follows. The remainder of the introduction discusses the related literature. Section 2 documents the heterogeneity in sensitivities across U.S. regions and how it is best explained by climate. Section 3 presents and estimates our simple structural model of adaptation. Section 4 calculates the effect of climate change based on our structural model. Section 5 concludes.

Literature review The growing literature on the economics of climate change, pioneered by Nordhaus (Nordhaus [1994], Nordhaus and Boyer [2000]), focuses primarily on the economy's effect on the climate and how policy should address the central pollution externality by taxing carbon or encouraging R\&D; see among recent examples, Acemoglu et al. [2016] and Golosov et al. [2014]. In contrast, our paper focuses solely on the propagation from climate to economy. Heal [2017] and Auffhammer [2018] are two recent surveys.

We build directly on the recent "new climate-economy literature", surveyed in Dell et al. [2014], which uses panel data to identify the economic impact of "weather shocks". Dell et al. [2012], Burke et al. [2015], and Kahn et al. [2019] use cross-country data to show that gross domestic product (G.D.P.) is negatively affected by higher than average temperature realizations, and that these effects are nonlinear and affect even wealthy countries. Deryugina and Hsiang [2014], Deryugina and Hsiang [2017], Colacito et al. [2019], Boldin and Wright [2015], Bloesch and Gourio [2015], Foote [2015], and Wilson [2017b] provide more evidence that weather affects the U.S. economy, often using detailed regional level data. ${ }^{3}$ A number of studies try to understand the different channels at work, including hours worked (Graff Zivin and Neidell [2014]), productivity (Heal and Park [2013]), farming (Deschenes and Greenstone [2007] or Schlenker and Roberts [2009]), or retail (Tran [2019]).

Finally, our paper is related to a growing literature on the effects of adaptation. One example is Barreca et al. [2016] who show that mortality became less sensitive to hot days over the last century because of diffusion of air conditioning. ${ }^{4}$

\footnotetext{
${ }^{3}$ The effect of weather or climate on output can also be observed in asset markets, as shown in Bansal et al. [2016a], Bansal et al. [2016b], Wilson [2017a], or Kruttli et al. [2019].

${ }^{4}$ See also Fried [2019] and Costinot et al. [2016].
} 


\section{The cross-section of temperature sensitivities}

The goal of this section is to characterize empirically the heterogeneity in sensitivity of economic activity to temperature at the county level in the United States. We build on the large recent climate-economy literature, and specifically on the study by Deryugina and Hsiang [2014]. We first review briefly the data used and the measurement of average effect of temperature, before turning to the heterogeneity. ${ }^{5}$

\subsection{Data}

We combine annual income data with daily weather data at the county level. The income statistics are compiled by the Bureau of Economic Analysis (B.E.A.) and provide a measure of a county's total personal income per capita. We construct our weather statistics using the U.S. historical climate network (US-HCN) database. US-HCN collects daily measures of average temperature for thousands of weather stations in the United States. We aggregate these measures at the county level by taking a simple average of all weather stations located within a county. Overall we obtain an unbalanced sample of 2,901 counties over the period 1969-2015, for a total of 65,537 observations.

\subsection{Average Sensitivity of Income to Temperature}

Following Deryugina and Hsiang [2014], we use the following specification to capture the possibly nonlinear effects of temperature on income. First, calculate a histogram of daily temperature for each county and each year. Denote $\operatorname{Bin}_{k, i, t}$ the number of days in year $t$ in county $i$ where temperature falls in bin $k=1 \ldots K$, where the bins are $3^{\circ} \mathrm{C}$ wide bands. Specifically, bin 1 range is $-15^{\circ} \mathrm{C}$ or less, bin 2 is $-15^{\circ} \mathrm{C} /-12^{\circ} \mathrm{C}, \ldots$, bin 15 is $24^{\circ} \mathrm{C} / 27^{\circ} \mathrm{C}$, bin 16 is $27^{\circ} \mathrm{C} / 30^{\circ} \mathrm{C}$, and bin 17 is $30^{\circ} \mathrm{C}$ and above. The baseline specification is

$$
\Delta \log Y_{i, t}=\alpha_{i}+\delta_{t}+\sum_{k=1}^{K} \beta_{k} \operatorname{Bin}_{k, i, t}+\varepsilon_{i, t}
$$

where $\Delta \log Y_{i, t}$ is the growth rate of income per capita in county $i$ in year $t, \alpha_{i}$ is a county fixed effect, and $\delta_{t}$ a year fixed effect. The central bin $\left(12^{\circ} \mathrm{C}-15^{\circ} \mathrm{C}\right)$ is omitted, providing a reference by which temperature deviations are evaluated.

This specification is appealing because the distribution of days across bins of temperature varies from year to year due to quasi-random weather fluctuations. With the inclusion of county fixed effects we are effectively comparing the growth rate of income in a county in two years that differ in their realized distribution of daily temperature.

The key result is that the coefficients $\beta_{k}$ are negative for the top bins, corresponding to the hottest days. An additional day in the bin $27^{\circ} \mathrm{C}-30^{\circ} \mathrm{C}$ or in the bin $30^{\circ} \mathrm{C}+$ reduces annual income

\footnotetext{
${ }^{5}$ Our online appendix contains additional results and robustness analysis.
} 
by about $0.04 \%$ relative to the omitted reference bin $\left(12^{\circ} \mathrm{C}-15^{\circ} \mathrm{C}\right)$. In the appendix, we report the full set of results, which reproduce closely those of Deryugina and Hsiang [2014]. If income is generated linearly across the year, one day corresponds to $1 / 365=0.27 \%$ of annual income; to generate a $0.04 \%$ decrease in annual income, a hot day must then reduce daily income by a quite large $15 \%$ (or $0.04 / 0.27$ ). ${ }^{6}$

There are a variety of channels through which temperature affects economic activity, some positive, others negative. As discussed in the introduction, previous research has highlighted that both productivity and hours worked are affected negatively by high temperatures. Even in the United States, many workers are not fully insulated from temperature during work, during their commute, or at home, affecting their hours and productivity. For instance, while offices are often air-conditioned, factories or warehouses may not be. Moreover, some sectors rely on outside activity, such as construction, farming, or some leisure activities, and temperature directly affects the productivity of these activities. There could also be local general equilibrium effects, for instance lower income of construction workers leads to lower spending restaurants. We do not try to disentangle these mechanisms in this paper - we focus on the macroeconomic, comprehensive effect as summarized by the estimate. ${ }^{7}$

\subsection{Heterogeneity in Sensitivity of Income to Temperature}

We now turn to our empirical contribution. Motivated by the results discussed in the previous section, we use interaction models of the form:

$$
\Delta \log Y_{i, t}=\alpha_{i}+\delta_{t}+\sum_{k=1}^{K}\left(\sum_{l=0}^{L} \beta_{k l} x_{i l}\right) \operatorname{Bin}_{k, i, t}+\varepsilon_{i, t},
$$

where $x_{i l}$ are $L$ county characteristics that might affect the sensitivity to temperature. (We focus on characteristics that are constant over time for simplicity.) We will use different versions of equation (2) corresponding to different characteristics $x_{i l}$ to make three points: (1) climate (the long-term average temperature) has a large effect on the sensitivity, (2) even when we control for other county characteristics, and (3) other characteristics have much smaller effects on the sensitivity than climate does.

We measure the sensitivity as the marginal effect of a hot day (defined here as $27^{\circ} \mathrm{C}+$ ) on annual income as implied from equation (2):

$$
M E H D_{i}=\sum_{l=0}^{L} \beta_{K l} x_{i l} .
$$

\footnotetext{
${ }^{6}$ Note, however, that income is not always generated linearly over the year; for instance a day too hot might kill crops, reducing significantly the entire annual income, and certainly more than $100 \%$ of a day.

${ }^{7}$ Two data limitations are worth mentioning. First, income is measured in nominal terms, for lack of local price indices. National inflation is captured by the time effect, but temperature may affect local inflation. The inflationadjusted income response is higher (lower) than our estimate if inflation rises (falls) with temperature.Second, firm profits are captured by this income measure only to the extent that they are distributed locally, generating a downward bias.
} 
Figure 1 depicts this marginal effect as a function of couty's characteristics, for nine alternative models which differ in the set of variables included in $x_{i l}$. The first row depicts the results of models that use only the long-run average county temperature, which we denote $\overline{T_{i}}$ : panel A shows a linear model, so $x_{i l}=\left\{1, \overline{T_{i}}\right\}$, panel B a quadratic model, $x_{i l}=\left\{1, \overline{T_{i}}, \bar{T}_{i}^{2}\right\}$, and panel $\mathrm{C}$ a cubic model $x_{i l}=\left\{1, \bar{T}_{i}, \bar{T}_{i}^{2}, \bar{T}_{i}^{3}\right\} .8$ Consistently across the three models, the sensitivity is negative and large in absolute values for colder places (low long-run average temperature) and shrinks towards zero as average temperature increases. ${ }^{9}$ There is substantial statistical uncertainty about the marginal effects for cold places (because cold places have few hot days). But the differences in the magnitudes are very large even focusing on counties with average temperature in the center of the distribution, say between $11^{\circ} \mathrm{C}$ and $18^{\circ} \mathrm{C}$, demonstrating our point (1). Panels B and $\mathrm{C}$ suggest significant nonlinearities, and the quadratic term is statistically significant at the $5 \%$ level - so from now on we use the quadratic model as benchmark.

One potential concern is that the long-run average temperature of a county might be correlated with other characteristics that affect the sensitivity to temperature, such as income, population, or the importance of farming for the local economy. The second row of figure 1 considers this possibility and adds as interaction variables, on top of the quadratic in long-run average temperature: in panel (D), the long-run average of income per capita (normalized by national income per capita), in panel (E), the long-run average of the farming share of income in the county; and in panel (F), both of these variables, plus the long-run average of log population (normalized by national population). The estimated marginal effects remain largely unchanged: the lower sensitivities in warmer places are not driven by these potential confounders. This demonstrates our point (2).

Finally, one may ask, quantitatively, what is the importance of these other, non-climate variables for the sensitivity, compared to average temperature? The third row shows three models that do not include average temperature, but only either population (in panel G), income (panel H), or the farming share (panel I), and including the square of each measure to allow for nonlinearities. In all cases, the effect, while meaningful, is substantially smaller than the effect of average temperature. This demonstrates our point (3).

Overall, we conclude that there is a wide heterogeneity in sensitivity to temperature across U.S. counties, and this heterogeneity is mostly explained by average temperature. ${ }^{10}$

\footnotetext{
${ }^{8}$ In the interest of keeping the graphs easy to read with a single scale, Panel C does not include standard errors.

${ }^{9}$ For each figure, the $\mathrm{x}$-axis ranges from the 5 th percentile to the 95 th percentile of the interaction variable, in that case long-run average temperature.

${ }^{10}$ Table 5 in appendix reports the coefficients and standard errors corresponding to each of these models, and shows that average temperature "drives out" the other variables. The appendix provides additional empirical results, in particular to show that similar results obtain if we use (i) alternative measures of climate, such as the frequency of hot days, (ii) other thresholds than $27^{\circ} \mathrm{C}$ for the warmest days, and (iii) alternative statistical models.
} 
A

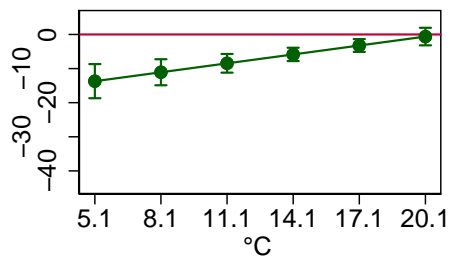

$\mathrm{D}$

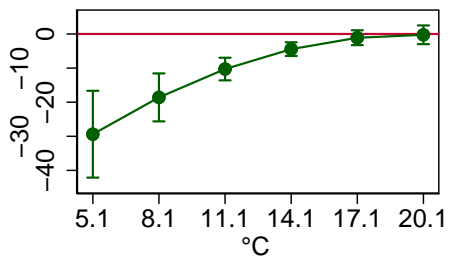

G

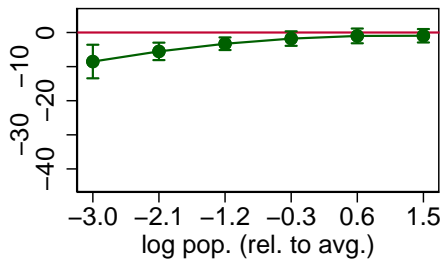

B

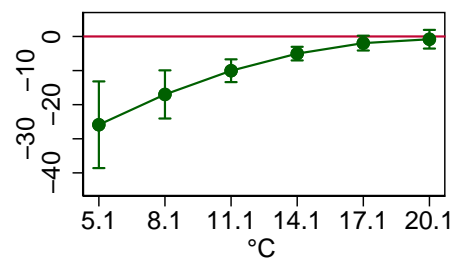

E

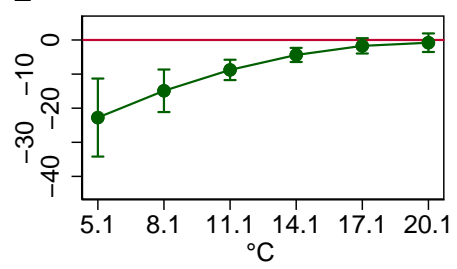

$\mathrm{H}$

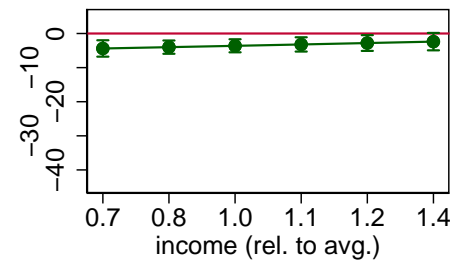

C

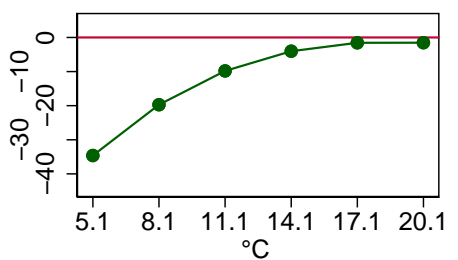

F

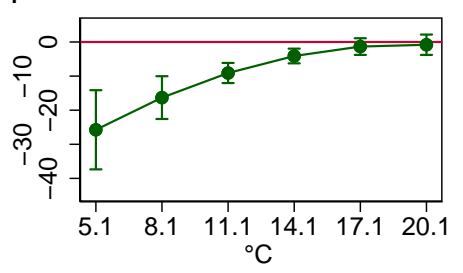

I

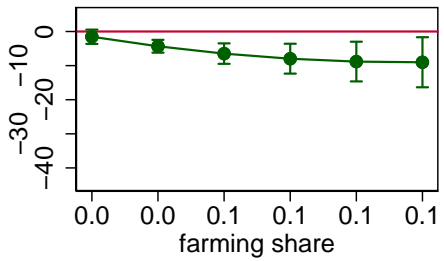

Figure 1: Marginal effect of a day above $27^{\circ} \mathrm{C}$ on annual income, as a function of the county's average temperature (panels A-F) or some other characteristic (panels G-I).

Note: The figure depicts the estimated marginal effect (in basis points) of an additional day in the $27^{\circ} \mathrm{C}$ plus bin, for different models of equation (2), together with plus and minus two standard error bands (doubleclustered by county and by NOAA region-year). Panels A-F plot the marginal effect as a function of average temperature, while panels G-I plot it as a function of some other county characteristics. Panel A: interaction is linear in average temperature; panel B: quadratic in average temperature; Panel C: cubic in average temperature; Panel D: quadratic in average temperature, plus average income (relative to national); Panel E: quadratic in average temperature, plus average farming share; Panel F: quadratic in average temperature, plus average population (in log, relative to national), average income (relative to national), and average farming share; Panel G: quadratic in average population (in log, relative to national) ; Panel H: quadratic in average income (relative to national); Panel I: quadratic in average farming share.

\section{A Simple Model of Adaptation}

This section presents and estimates a simple model of adaptation. In the model, high temperatures reduce output, but agents can decide to invest in adaptation, which reduces the sensitivity of output to temperature. In the first subsection, we present the model, and in the second subsection, we estimate the model using indirect inference. 


\subsection{Setup}

The economy is made up of a finite number of independent closed economies (counties): there is neither trade nor population migration. Each county's production output is affected by the local temperature, but the effect of temperature depends on the level of adaptation. Specifically, each county makes a once-and-for-all decision at time 0 of how much to spend on adaptation. Thereafter, output is generated according to:

$$
Y_{i t}=f\left(T_{i t}, k_{i}\right)
$$

where $Y_{i t}$ is output in county $i$ at time $t, T_{i t}$ is temperature, and $k_{i}$ is spending on adaptation. We measure $k_{i}$ in percentage of output, and in annuity terms, so $k_{i}$ is the per period share of output paid in perpetuity. The function $f$ bundles the production and adaption technology, and will capture the empirical regularity documented in Section 2, that hot days reduce income. As we will see, the slope of this relation varies across counties because they decide on different adaptation $k_{i}{ }^{11}$ Temperature $T_{i t}$ is assumed to be iid over time and drawn from a constant countyspecific cumulative distribution function $F_{i}{ }^{12}$ This temperature distribution is the only exogenous difference across counties in our model.

We abstract from saving or borrowing, hence consumption of county $i$ equals output, net of adaptation costs:

$$
C_{i t}=Y_{i t}\left(1-k_{i}\right)
$$

We assume standard expected utility preferences:

$$
(1-\beta) E \sum_{t=0}^{\infty} \beta^{t} u\left(C_{i t}\right),
$$

Given our i.i.d. assumption, this can be rewritten as:

$$
V\left(k_{i} ; F_{i}\right)=E u\left(\left(1-k_{i}\right) f\left(T_{i t}, k_{i}\right)\right),
$$

\footnotetext{
${ }^{11}$ The function $f$ can be microfounded, for instance using a stochastic growth model where productivity and labor disutility vary with temperature. To spell out a simple example, assume a production function

$$
Y_{i t}=A_{i t} N_{i t}^{\alpha}
$$

where $A_{i t}$ is total factor productivity and $N_{i t}$ is labor supplied, and $A_{i t}$ depend on temperature $T_{i t}$ and adaptation $k_{i}$. The per-period utility function is

$$
u\left(C_{i t}, N_{i t}\right)=\frac{C_{i t}^{1-\sigma}}{1-\sigma}-\xi_{i t} \frac{N_{i t}^{1+\psi}}{1+\psi},
$$

where the utility cost of working $\xi_{i t}$ depends on $T_{i t}$ and $k_{i}$. This model can be solved in closed form solution: output is a function of $A_{i t}$ and $\xi_{i t}$, and hence ultimately of temperature $T_{i t}$. This model hence leads to the equation (4) in the text. We do not attempt to estimate this more detailed economic model, because of data limitations (e.g. on labor input and productivity), but this would be an interesting extension.

${ }^{12}$ Of course, the iid assumption is not true at the daily frequency, but taking this into account would not affect our results since the model has no inter-temporal linkages. Similarly, the absence of cross-county linkages makes the correlation of temperature across counties is immaterial.
} 
where the expectation is taken over $T_{i t}$, drawn according to $F_{i}$. Higher adaptation reduces consumption directly due to the adaptation costs, but increases available output by reducing the losses due to high temperature. The optimal adaptation decision balances these costs and benefits:

$$
k_{i}^{*}=\arg \max _{k_{i}} V\left(k_{i} ; F_{i}\right) .
$$

Overall the model takes as inputs the county-specific distribution of temperature $F_{i}$ and the common across counties utility and production/adaptation functions $u$ and $f$, and delivers a county-specific adaptation choice $k_{i}$ and output stochastic process $Y_{i t}$.

\subsection{Functional forms and solution method}

This section presents the functional forms used for our baseline results; the appendix shows how our results change with alternative functional forms. First, we assume utility is $\log , u(c)=$ $\log (c)$. Second, motivated by the empirical patterns of section 2, we assume that output falls with temperature above a threshold $\bar{T}$ :

$$
\begin{aligned}
\log f\left(T_{i t}, k_{i}\right) & =b_{0} \text { if } T_{i t}<\bar{T} \\
& =b_{0}-\overline{b_{1}} e^{-k / \theta}\left(T_{i t}-\bar{T}\right) \text { if } T_{i t} \geq \bar{T}
\end{aligned}
$$

Here,

- $b_{0}$ is the baseline productivity, assumed constant over time and across counties (given our empirical approach, which removes county and time fixed effects, this simplification is without loss of generality);

- $\bar{T}$ is the temperature threshold past which productivity starts to fall;

- $\overline{b_{1}}$ is the rate at which output falls with each degree above $\bar{T}$;

- $\theta$ measures the cost of adaptation, i.e. how spending on adaptation $k$ translates into sensitivity reduction $e^{-k / \theta}$.

\subsection{Model estimation}

To estimate the model, we fit the heterogeneity in the observed sensitivities to temperature across the United States.

Specifically, our model has only three parameters $\bar{T}, \overline{b_{1}}$ and $\theta$. Based on the evidence above, we set the threshold temperature $\bar{T}$ to $26^{\circ} \mathrm{C} .{ }^{13}$ To estimate the other two parameters $\overline{b_{1}}$ and $\theta$, we use indirect inference (Gourieroux et al. [1993], Smith [1993]). We choose as target moments the

\footnotetext{
${ }^{13}$ See figure 1 in appendix.
} 
sensitivity of income to hot days, i.e. the coefficients $\gamma_{q}$ from the panel regression:

$$
\Delta \log Y_{i, t}=\alpha_{i}+\delta_{t}+\gamma_{q} H D_{i, t}+\varepsilon_{i, t}
$$

where $H D_{i t}$ is the number of hot days in county $i$ in year $t$. We estimate this regression separately for each quintile $q$ of counties, sorted by their average annual number of hot days. This evidence, which is similar to the interaction model of section 2 , is presented in the first row of table 1 : counties in the higher quintiles (more hot days on average) have a lower sensitivity to hot days.

We find numerically the two parameters $\overline{b_{1}}$ and $\theta$ that minimize the distance ${ }^{14}$ between the vector of data moments $\gamma=\left(\gamma_{1}, \gamma_{2}, \gamma_{3}, \gamma_{4}, \gamma_{5}\right)$ and the vector of model moments $\widetilde{\gamma}$. The model moments are obtained, for each potential $\left(\overline{b_{1}}, \theta\right)$ by solving the model numerically, simulating it, and estimating the same regression (11) on simulated data. ${ }^{15}$

\begin{tabular}{lccccccc}
\hline Source & Quintile1 & Quintile2 & Quintile3 & Quintile4 & Quintile5 & Jstat & pval \\
\hline Data & -0.117 & -0.091 & -0.065 & -0.042 & -0.008 & $\cdot$ & $\cdot$ \\
SE & 0.075 & 0.048 & 0.020 & 0.009 & 0.009 &. &. \\
Baseline & -0.088 & -0.096 & -0.072 & -0.035 & -0.017 & 1.881 & 0.598 \\
\hline
\end{tabular}

Table 1: The table reports the targeted moments, i.e. the $\gamma_{q}$ from equation 11, with estimated standard errors, together with the model moments at the estimated structural parameters.

The results of this procedure are presented in table 1. First, the model matches well the pattern that sensitivities are lower in warmer places. Also, because we have 5 target moments and only 2 parameters, the model is overidentified; the J-statistic is 1.88, and the model is not rejected (pval $=59 \%$ ). Second, we estimate $\overline{b_{1}}=0.161$ : with no adaptation, an additional degree above $26^{\circ} \mathrm{C}$ reduces income by $0.161 \mathrm{log}$ points. This is a large effect, which is required to fit the data. But note that this coefficient is significantly smaller with adaptation. The cost of adaptation $\theta$ is fairly low at 0.013 , hence reducing the sensitivity by $50 \%$ costs $k=-\log (2) \theta=0.9 \%$ of output, and reducing the sensitivity by $90 \%$ costs $3.0 \%$ of output.

\section{Implications for climate change}

We now use our structural model to predict the effect of climate change on income. We first describe our methodology, then our results.

\subsection{Methodology}

Our calculations require two inputs: a climate forecast by county over the next century; and an economic model that maps temperature into income.

\footnotetext{
${ }^{14}$ The distance is calculated using as weighting matrix the inverse of the variance of the data estimates.

${ }^{15}$ To solve the model for parameter $\left(\overline{b_{1}}, \theta\right)$, we first calculate the optimal $k_{i}$ given the county's distribution $F_{i}$ and the parameters. There is no closed form solution for this model; we solve it numerically after discretizing the distribution $F_{i}$.
} 


\subsubsection{Climate forecast}

Following Hsiang et al. [2017], we base our climate projections on Rasmussen et al. [2016], which provides county-specific projections from the current period to 2099 corresponding to four different possible greenhouse gas emissions scenarios. RCP 2.6 represents high greenhouse gas mitigation, RCP 4.5 moderate mitigation, RCP 6.0 low mitigation and finally RCP 8.5 is a "business-as-usual" emission scenario. For each RCP scenario, these projections approximate the future distribution of temperature by weighting about 120 different climate models (using the Surrogate/Model Mixed Ensemble method projection). We calculate the effect of a RCP scenario by averaging the changes implied by each underlying climate model.

\subsubsection{Economic model}

We will contrast two specifications of our structural model of section 3 . In the first case, we assume that each county's adaptation level $k_{i}$ remains fixed at the level currently estimated. In the second case, we assume that adaptation is endogenously re-optimized given the new climate. We call these the fixed and endogenous adaptation cases respectively.

\subsection{Results}

Table 2 presents, for each RCP scenario, the estimated losses across U.S. counties under both the fixed and endogenous adaptation assumptions. We report the effect on income and consumption (income net of adaptation costs). We draw four main conclusions from this table, and the associated figures 2 (a histogram of losses) and 3 (a map of losses).

Result 1: Adaptation following climate change reduces median losses This conclusion is of course qualitatively preordained; counties can do no worse than keeping their current adaptation level, so by optimizing it they reduce their losses. But the magnitudes of the reductions in losses is substantial. For instance, in the RCP 6.0 scenario, the median consumption loss under fixed adaptation is $-2.92 \%$, but only $-1.80 \%$ under endogenous adaptation. ${ }^{16}$ The differences are even larger when considering median output (gross of adaptation costs), which actually increases by $0.27 \%$ under endogenous adaptation because places that are initially cold decide to adapt and hence do not lose as much income on hot days as they used to.

Result 2: Adaptation following climate change reduces the dispersion of losses Perhaps as important from a welfare point of view, the dispersion in losses is also reduced once re-optimization of adaptation is taken into account. Focusing again on the RCP 6.0 scenario, the standard deviation of losses is $1.48 \%$ with fixed adaptation and only $0.65 \%$ with endogenous adaptation.

The economic mechanism is that counties that have the most to lose from climate change will benefit the most from re-optimizing adaptation; as a result, the left tail of outcomes is strongly truncated. For instance, the 10th percentile of losses is $-4.97 \%$ under fixed adaptation and only $-2.60 \%$ with endogenous adaptation. This reduction in dispersion is clear in figure 2.

\footnotetext{
${ }^{16}$ These losses are permanent, i.e. hold for each year in perpetuity.
} 
Result 3: Adaptation is more important the larger the change in climate. The difference between the fixed and endogenous adaptation model is larger, the larger is the change in climate. This is intuitive: if climate hardly changes, there is little gain to re-optimizing adaptation. ${ }^{17}$ The magnitudes, however, are impressive. The difference between median losses under fixed vs. endogenous adaptation is $0.14 \%$ in the RCP 2.6 scenario, and rises to $0.82 \%, 1.12 \%$, and finally $3.45 \%$ in the RCP 8.5 scenario. The difference at the 10 th percentile increase even further, from $0.3 \%$ to $6.42 \%$.

Result 4: Adaptation (both current and future) affects substantially the location of losses Figure 3 maps the estimated losses for three models, again for the RCP 6.0 scenario; on top of the fixed adaptation and endogenous adaptation cases, this figure also incorporates an additional "uniform sensitivity" case. This case is obtained by setting $\theta=\infty$ (i.e. infinite cost of adaptation) in our model, rather than using the estimated value. ${ }^{18}$ This approach amounts to assuming a constant sensitivity across U.S. regions, which is furthermore invariant to climate change. ${ }^{19}$ We find it interesting to contrast our results with this approach, which has been used in some influential papers, such as Hsiang et al. [2017].

First, note that in this "uniform sensitivity" case, the largest losses in the U.S. are born in the South and Southeast regions. This makes intuitive sense, as these regions see the largest increase in the number of hot days. By contrast, under the fixed adaptation model, the largest losses are borne by states in the NOAA Central region, roughly the Midwest. To understand why, note that the losses reflect two factors: (1) the currently measured sensitivities and (2) the increase in the number of hot days post-climate change. Because the sensitivities are much lower in the South, we obtain that losses are smaller there than in the Central region, even though the increase in the number of hot days is larger in the South. In contrast, the uniform sensitivity model assumes that the South is highly sensitive to hot days (an implication that is soundly rejected in the data).

In the endogenous adaptation case, the regional distribution is similar, but the Central region in particular undertakes significant additional adaptation to reduce its losses. Comparing the maps confirms the lower and less dispersed losses in the endogenous case discussed in Results 1 and 2 above. Overall, the differences in regional implications are extreme: the correlation between the predicted losses in the uniform sensitivity model and in the fixed (resp. endogenous) adaptation model is only 0.09 (resp. -0.09).

\subsection{Extrapolation}

We discussed in the introduction some caveats to our approach; here we want to focus on extrapolation. By definition, the county that is currently the hottest in the U.S. will become warmer than any county in our data after climate change. To infer what will happen to that county requires some extrapolation. But how much extrapolation is actually required?

\footnotetext{
${ }^{17}$ Indeed, since adaptation is initially chosen optimally, the benefit to changing adaptation is second-order.

${ }^{18}$ We re-estimate the parameter $\overline{b_{1}}$ using our indirect inference approach.

${ }^{19}$ While we derive this approach from our structural model, it can also be obtained in a purely statistical way using a pooled panel regression such as equation (1).
} 


\begin{tabular}{lcccccc}
\hline \hline & & & & & & \\
& Median & Std.Dev. & p10 & p25 & p75 & p90 \\
\hline Panel A: $\boldsymbol{R C P}$ 2.6 & & & & & & \\
Fixed adapt. C,Y & -0.91 & 0.52 & -1.62 & -1.26 & -0.63 & -0.30 \\
Endogenous adapt. C & -0.77 & 0.39 & -1.32 & -1.06 & -0.55 & -0.30 \\
Endogenous adapt. Y & 0.33 & 1.30 & -0.58 & -0.39 & 1.88 & 2.72 \\
Panel B: $\boldsymbol{R C P}$ 4.5 & & & & & & \\
Fixed adapt. C,Y & -2.33 & 1.22 & -4.07 & -3.27 & -1.63 & -1.04 \\
Endogenous adapt. C & -1.51 & 0.58 & -2.24 & -1.93 & -1.13 & -0.85 \\
Endogenous adapt. Y & 0.29 & 1.38 & -0.82 & -0.62 & 1.86 & 2.71 \\
Panel C: $\boldsymbol{R C P}$ 6.0 & & & & & & \\
Fixed adapt. C,Y & -2.92 & 1.48 & -4.97 & -4.02 & -2.05 & -1.37 \\
Endogenous adapt. C & -1.80 & 0.65 & -2.60 & -2.27 & -1.35 & -1.04 \\
Endogenous adapt. Y & 0.27 & 1.41 & -0.92 & -0.70 & 1.85 & 2.70 \\
Panel D: $\boldsymbol{R C P}$ 8.5 & & & & & & \\
Fixed adapt. C,Y & -6.17 & 2.72 & -10.03 & -8.37 & -4.25 & -3.08 \\
Endogenous adapt. C & -2.72 & 0.79 & -3.61 & -3.33 & -2.06 & -1.70 \\
Endogenous adapt. Y & 0.24 & 1.47 & -1.10 & -0.86 & 1.83 & 2.69 \\
\hline \hline
\end{tabular}

Table 2: The table reports cross-county statistics of predicted income and consumption (i.e., income net of adaptation) losses, for each climate change scenario (RCP 2.6 through RCP 8.5), for two model variants: (i) assuming that adaptation is fixed at the currently estimated value; (ii) assuming that adaptation is endogenously re-optimized after climate change.

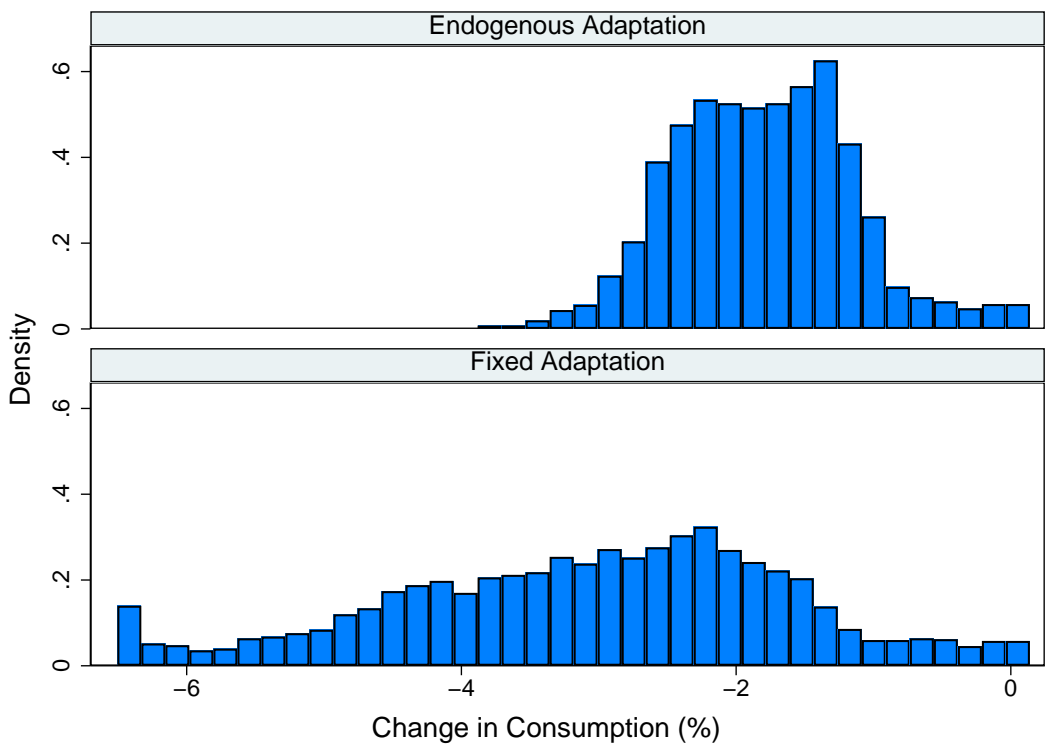

Figure 2: Distribution of predicted income losses across U.S. counties for the RCP 6.0 scenario. Top panel: endogenous adaptation; bottom panel: fixed adaptation.

Consider first the "extensive margin" of extrapolation: for some counties, the average number of hot days per year is greater, post-climate change, than is currently observed in any county. This extrapolation is, strictly speaking, limited: for instance, in the RCP 6.0 scenario, this affects only $0.2 \%$ of counties. However, in a broader sense, it is more significant: $8.5 \%$ counties have more hot 
days, post climate change, than the hottest $1 \%$ of U.S. counties do today. This suggests that our results are somewhat sensitive to our inference of adaptation from this relatively small sample of hot U.S. counties. On the "intensive margin", higher temperature may bring very hot days, yet our model assumes that the economic loss is log-linear above $\bar{T}$. In the appendix, we show that this functional form holds up rather well, but there is significant statistical uncertainty.

\section{Conclusion}

Climate change will lead to large economic losses. But the extent of the losses will depend critically on adaptation. This paper proposes a simple methodology that combines the well-identified estimates of the recent "climate-economy" literature with a structural model of adaptation to estimate the adaptation technology. We find that adaptation reduces both the first moment and the second moment (dispersion) of income losses, particularly when climate change is substantial, and changes dramatically the location of losses.

\section{References}

Daron Acemoglu, Ufuk Akcigit, Douglas Hanley, and William Kerr. Transition to clean technology. Journal of Political Economy, 124(1):52-104, 2016.

Maximilian Auffhammer. Quantifying economic damages from climate change. Journal of Economic Perspectives, 32(4):33-52, 2018.

Ravi Bansal, Dana Kiku, and Marcelo Ochoa. Price of long-run temperature shifts in capital markets. Technical report, National Bureau of Economic Research, $2016 \mathrm{a}$.

Ravi Bansal, Marcelo Ochoa, and Dana Kiku. Climate change and growth risks. Technical report, National Bureau of Economic Research, 2016b.

Alan Barreca, Karen Clay, Olivier Deschenes, Michael Greenstone, and Joseph S Shapiro. Adapting to climate change: The remarkable decline in the us temperature-mortality relationship over the twentieth century. Journal of Political Economy, 124(1):105-159, 2016.

Justin Bloesch and Francois Gourio. The effect of winter weather on us economic activity. Economic Perspectives, Federal Reserve Bank of Chicago, 2015.

Michael Boldin and Jonathan H. Wright. Weather-adjusting economic data. Brookings Papers on Economic Activity, pages 227-260, 2015.

Marshall Burke, Solomon M Hsiang, and Edward Miguel. Global non-linear effect of temperature on economic production. Nature, 527(7577):235-239, 2015. 
Riccardo Colacito, Bridget Hoffmann, and Toan Phan. Temperature and growth: A panel analysis of the united states. Journal of Money, Credit and Banking, 51(2-3):313-368, 2019.

Arnaud Costinot, Dave Donaldson, and Cory Smith. Evolving comparative advantage and the impact of climate change in agricultural markets: Evidence from 1.7 million fields around the world. Journal of Political Economy, 124(1):205-248, 2016.

Melissa Dell, Benjamin F Jones, and Benjamin A Olken. Temperature shocks and economic growth: Evidence from the last half century. American Economic Journal: Macroeconomics, 4(3):66-95, 2012.

Melissa Dell, Benjamin F Jones, and Benjamin A Olken. What do we learn from the weather? the new climate-economy literature. Journal of Economic Literature, 52(3):740-98, 2014.

Tatyana Deryugina and Solomon Hsiang. The marginal product of climate. Technical report, National Bureau of Economic Research, 2017.

Tatyana Deryugina and Solomon M Hsiang. Does the environment still matter? daily temperature and income in the united states. Technical report, National Bureau of Economic Research, 2014.

Olivier Deschenes and Michael Greenstone. The economic impacts of climate change: evidence from agricultural output and random fluctuations in weather. American Economic Review, 97(1): 354-385, 2007.

Christopher L Foote. Did abnormal weather affect us employment growth in early 2015? 2015.

Stephie Fried. Seawalls and stilts: A quantitative macro study of climate adaptation. Working Paper, 2019.

Mikhail Golosov, John Hassler, Per Krusell, and Aleh Tsyvinski. Optimal taxes on fossil fuel in general equilibrium. Econometrica, 82(1):41-88, 2014.

Christian Gourieroux, Alain Monfort, and Eric Renault. Indirect inference. Journal of applied econometrics, 8(S1):S85-S118, 1993.

Joshua Graff Zivin and Matthew Neidell. Temperature and the allocation of time: Implications for climate change. Journal of Labor Economics, 32(1):1-26, 2014.

Geoffrey Heal. The economics of the climate. Journal of Economic Literature, 55(3):1046-63, 2017.

Geoffrey Heal and Jisung Park. Feeling the heat: Temperature, physiology \& the wealth of nations. Technical report, National Bureau of Economic Research, 2013.

Solomon Hsiang, Robert Kopp, Amir Jina, James Rising, Michael Delgado, Shashank Mohan, DJ Rasmussen, Robert Muir-Wood, Paul Wilson, Michael Oppenheimer, et al. Estimating economic damage from climate change in the united states. Science, 356(6345):1362-1369, 2017. 
Matthew E Kahn, Kamiar Mohaddes, Ryan NC Ng, M Hashem Pesaran, Mehdi Raissi, and JuiChung Yang. Long-term macroeconomic effects of climate change: A cross-country analysis. Technical report, National Bureau of Economic Research, 2019.

Mathias Kruttli, Brigitte Roth Tran, and Sumudu W Watugala. Pricing poseidon: extreme weather uncertainty and firm return dynamics. 2019.

William D Nordhaus. Managing the global commons: the economics of climate change, volume 31. MIT press Cambridge, MA, 1994.

William D Nordhaus and Joseph Boyer. Warming the world: economic models of global warming. MIT press Cambridge, MA, 2000.

Robert S Pindyck. Climate change policy: What do the models tell us? Journal of Economic Literature, 51(3):860-72, 2013.

Brad Plumer and Nadja Popovich. As climate changes, southern states will suffer more than others. The New York Times, June 29, 2017.

DJ Rasmussen, Malte Meinshausen, and Robert E Kopp. Probability-weighted ensembles of us county-level climate projections for climate risk analysis. Journal of Applied Meteorology and Climatology, 55(10):2301-2322, 2016.

Wolfram Schlenker and Michael J Roberts. Nonlinear temperature effects indicate severe damages to us crop yields under climate change. Proceedings of the National Academy of sciences, 106(37): 15594-15598, 2009.

Anthony Smith. Estimating nonlinear time-series models using simulated vector autoregressions. Journal of Applied Econometrics, 8(S1):S63-S84, 1993.

Brigitte Roth Tran. Sellin'in the rain: Adaptation to weather and climate in the retail sector. 2019.

Daniel J Wilson. Clearing the fog: The predictive power of weather for employment reports and their asset price responses. Federal Reserve Bank of San Francisco Working Paper, 2017a.

Daniel J Wilson. The impact of weather on local employment: Using big data on small places. Federal Reserve Bank of San Francisco, 2017b. 


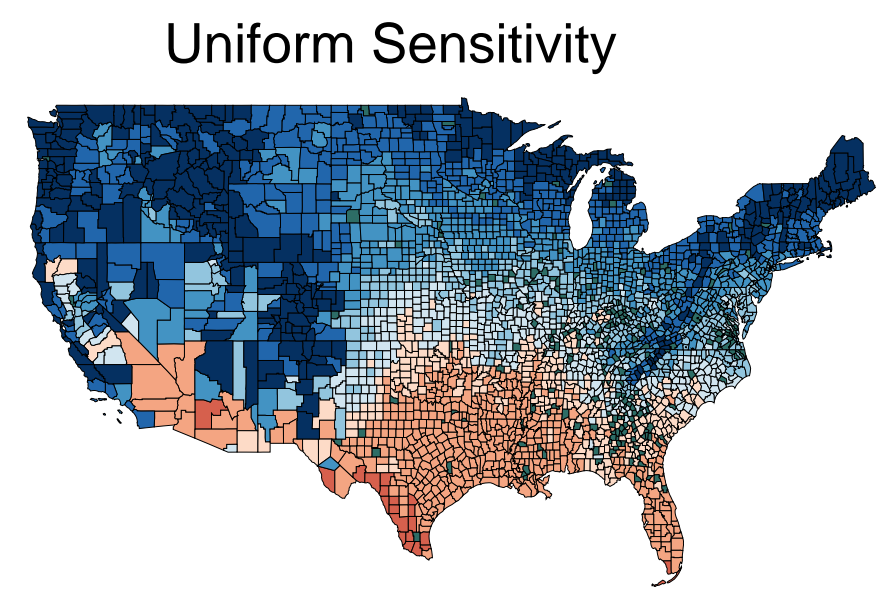

Fixed Adaptation
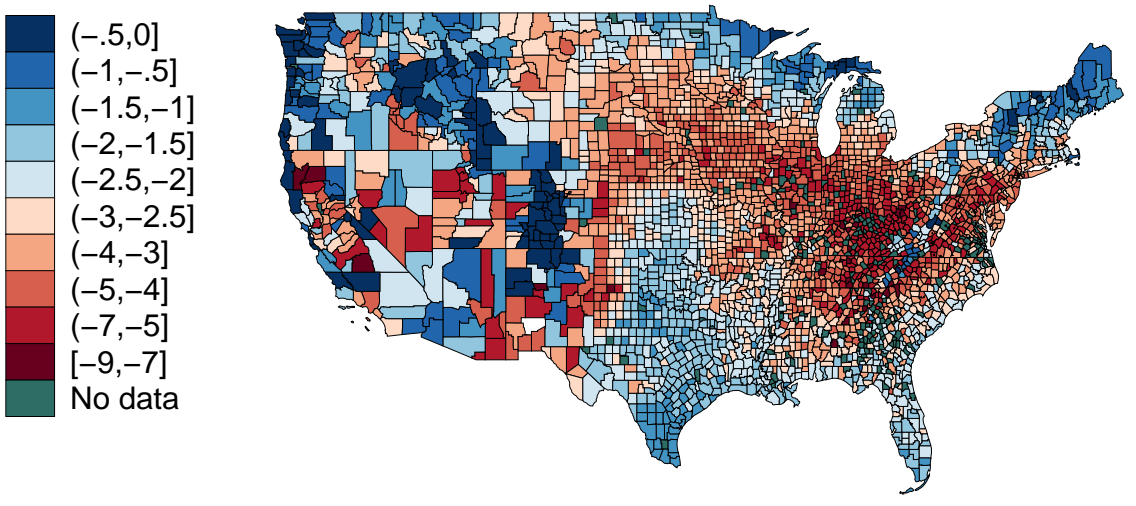

\section{Endogenous Adaptation}

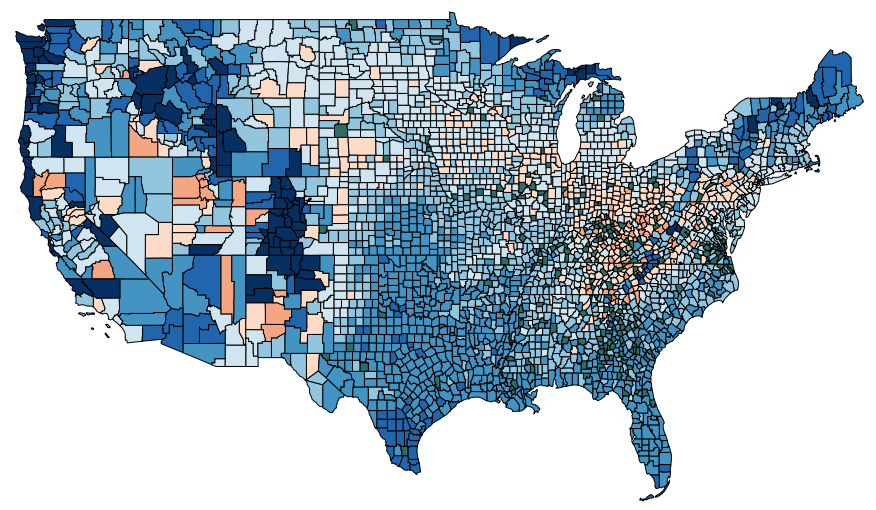

Figure 3: Predicted consumption losses for the RCP 6.0 scenario.

The figure displays the predicted decline in consumption (income net of adaptation costs) for each U.S. county in the RCP 6.0 scenario, for three different models. Top panel: uniform sensitivity; middle panel: fixed adaptation; bottom panel: endogenous adaptation. 